\title{
BSDEs with random terminal time and semilinear elliptic PDEs in divergence form
}

by

\author{
Andrzej Rozkosz (Toruń)
}

\begin{abstract}
We study connections between Sobolev space solutions of the Dirichlet problem for semilinear second order elliptic equations in divergence form and solutions of backward stochastic differential equations with random terminal time.
\end{abstract}

1. Introduction. In Peng [9] and Darling and Pardoux [4] (see also the expository paper by Pardoux [8] and the references given therein) connections between solutions of backward stochastic differential equations (BSDEs) with random terminal time and viscosity solutions of the Dirichlet problem for semilinear second order elliptic equations in non-divergence form are investigated. In the present paper we are interested in finding connections between Sobolev space solutions of the semilinear Dirichlet problem and solutions of BSDEs with random terminal time in the case when the forward driving process is a diffusion generated by a uniformly elliptic divergence form operator with measurable coefficients. We emphasize that it is not a semimartingale generally.

To be more precise, let us fix a bounded domain (non-empty, open, connected set) $D$ in $\mathbb{R}^{d}$ and consider the differential operator $A$ of the form

$$
A=\frac{1}{2} \sum_{i, j=1}^{d} D_{j}\left(a^{i j}(x) D_{i}\right)+\sum_{i=1}^{d} b^{i}(x) D_{i}
$$

where $a: D \rightarrow \mathbb{R}^{d} \otimes \mathbb{R}^{d}$ and $b: D \rightarrow \mathbb{R}^{d}$ are measurable functions such that for some $0<\lambda \leq \Lambda$,

$$
\lambda|\xi|^{2} \leq \sum_{i, j=1}^{d} a^{i j}(x) \xi_{i} \xi_{j} \leq \Lambda|\xi|^{2}, \quad a^{i j}(x)=a^{j i}(x), \quad|b(x)| \leq \Lambda
$$

for $x \in D$ and $\xi \in \mathbb{R}^{d}$. By putting $a^{i j}=\delta_{j}^{i}, b^{i}=0$ outside $D$ we can and will

2000 Mathematics Subject Classification: Primary 60H30; Secondary 35J60.

Research supported by State Committee for Scientific Research (KBN) under grant 0253 P03 200019. 
assume that $a, b$ are defined and satisfy $(1.2)$ in all $\mathbb{R}^{d}$. Let $p(\cdot, \cdot, \cdot)$ denote a weak fundamental solution for $A$ and let $\mathbb{X}=\left\{\left(X, P_{x}\right) ; x \in \mathbb{R}^{d}\right\}$ be a Markov process for which $p$ is a transition density function, that is,

$$
P_{x}\left(X_{0}=x\right)=1, \quad P_{x}\left(X_{t} \in \Gamma\right)=\int_{\Gamma} p(t, x, y) d y, \quad t \geq 0,
$$

for any Borel $\Gamma \subset \mathbb{R}^{d}$ (see [16]). From [10, Theorem 3.4] it follows that for each starting point $x \in \mathbb{R}^{d}$ the canonical process $X$ admits a unique decomposition of the form

$$
X_{t}-X_{0}=M_{t}+A_{t}, \quad t \geq 0, P_{x^{-} \text {a.s. },}
$$

where $M$ is a continuous martingale additive functional (in the strict sense) of $\mathbb{X}$ and $A$ is a continuous additive functional (in the strict sense) of $\mathbb{X}$ of zero quadratic variation. Moreover, the co-variation process of $M=$ $\left(M^{1}, \ldots, M^{d}\right)$ is given by

$$
\left\langle M^{i}, M^{j}\right\rangle_{t}=\int_{0}^{t} a^{i j}\left(X_{s}\right) d s, \quad t \geq 0 .
$$

Suppose that $D$ is regular for $A$. For given $\varphi: \partial D \rightarrow \mathbb{R}$ and $f: D \times \mathbb{R} \times \mathbb{R}^{d}$ $\rightarrow \mathbb{R}$ consider the semilinear Dirichlet problem

$$
A u=-F_{u},\left.\quad u\right|_{\partial D}=\varphi
$$

where

$$
F_{u}(x)=f(x, u(x),(\sigma \nabla u)(x)), \quad x \in D,
$$

and $\sigma$ is the symmetric square root of $a$. In this paper we show that under some natural assumptions on $\varphi$ and $f$ there exists a bounded continuous solution $u$ of (1.5) in the Sobolev space $W_{2}^{1}$ and for each $x \in D$ there also exists a unique solution $\left(Y^{x}, Z^{x}\right)$ of the BSDE

$$
\begin{aligned}
Y_{t}^{x}= & \varphi\left(X_{\tau}\right)+\int_{t \wedge \tau}^{\tau} f\left(X_{s}, Y_{s}^{x}, Z_{s}^{x}\right) d s \\
& -\int_{t \wedge \tau}^{\tau}\left\langle Z_{s}^{x}, \sigma^{-1}\left(X_{s}\right) d M_{s}\right\rangle, \quad t \geq 0, P_{x^{-}} \text {a.s. }
\end{aligned}
$$

where

$$
\tau=\inf \left\{t \geq 0:\left|X_{t}\right| \notin D\right\} .
$$

Moreover, for each $x \in D$ we have

$$
\left(Y_{t}^{x}, Z_{t}^{x}\right)=\left(u\left(X_{t \wedge \tau}\right),(\sigma \nabla u)\left(X_{t \wedge \tau}\right)\right), \quad t \geq 0, P_{x^{-}} \text {a.s. }
$$

From (1.8) it follows in particular that $Y_{0}^{x}=u(x) P_{x}$-a.s. for $x \in D$ and $Z_{0}^{x}=(\sigma \nabla u)(x) P_{x}$-a.s. for a.e. $x \in D$, which yields a stochastic representation of $u$ and its gradient. 
For regular $a$ (i.e. if $A$ is a non-divergence form operator and $X$ is an Itô process under $P_{x}$ ), a representation similar to (1.8) was proved in Darling and Pardoux [4] under assumptions on $\varphi$ and $f$ similar to those in the present paper, and in Peng [9] under more restrictive assumptions. Let us also mention that the Cauchy problem for the semilinear parabolic equation $D_{t} u+A u=F_{u}$ with $A$ given by (1.1) is investigated in Bally, Pardoux and Stoica [1], Rozkosz [11] and Stoica [15], and the mixed boundary problem in Lejay [7]. Let us stress, however, that in [1] a representation of the form $(1.8)$ is obtained for quasi-every starting point $x \in D$, whereas in $[7,15]$ only for a.e. $x \in D$.

As for proofs, we would like to point out only one aspect. Roughly speaking, the main difficulty in proving (1.5)-(1.8) for every (and not quasi- or almost every) starting point is that we have to show $P_{x}$-integrability of the quadratic variation of the martingale part of (1.6) (or the martingale part of $u\left(X_{\cdot \wedge \tau}\right)$ in its decomposition of the form (1.3)). From (1.4), (1.6) it is therefore clear that to the requirement that the solution $u$ of (1.5) belongs to the space $C\left([0, \infty) ; \mathbb{R}^{d}\right) \cap W_{2}^{1}$ a condition on the integrability of $\nabla u$ must be added. Therefore in the present paper we consider for each $x \in D$ a Sobolev space $\mathcal{W}_{\alpha}(x)$ with weight, the weight being the $\alpha$-Green function for $A$ in $D$ with suitably chosen $\alpha \geq 0$ (see Section 2). Using stochastic calculus we derive a sort of "energy estimates" in the space $\mathcal{W}_{\alpha}(x)$. The crucial fact is that these estimates do not involve any regularity assumptions about $a$ and allow one to prove existence of a unique continuous solution $u \in W_{2}^{1}$ of $(1.5)$ such that $u \in \mathcal{W}_{\alpha}(x)$ for every $x \in D$. This is enough for our purposes, because from the definition of $\mathcal{W}_{\alpha}(x)$ it follows in particular that $E_{x} \int_{0}^{\tau}\left|\nabla u\left(X_{t}\right)\right|^{2} d t<\infty$ if $u \in \mathcal{W}_{\alpha}(x)$. As far as we know, the idea of using the spaces $\mathcal{W}_{\alpha}(x)$ for investigation of the problem (1.5) is new. Notice, however, that an idea similar in spirit has appeared in [11].

Notation. $\Omega=C\left([0, \infty) ; \mathbb{R}^{d}\right)$ is the space of continuous $\mathbb{R}^{d}$-valued functions on $[0, \infty)$ equipped with the topology of uniform convergence on compact sets. $X$ is the canonical process on $\Omega, X_{t}^{\tau}=X_{t \wedge \tau}, t \geq 0$. By $E_{x}$ (resp. $E_{x}^{n}$ ) we denote expectation with respect to $P_{x}$ (resp. $\left.P_{x}^{n}\right)$, by $\mathcal{L}\left[Y \mid P_{x}\right]$ (resp. $\mathcal{L}\left[Y \mid P_{x}^{n}\right]$ ) the law of the process $Y$ under $P_{x}$ (resp. $\left.P_{x}^{n}\right)$, and by “ $\Rightarrow$ " convergence in law.

$D_{i}=\partial / \partial x^{i}$ is the partial derivative in the distribution sense, and $\nabla=$ $\left(D_{1}, \ldots, D_{d}\right)$. By $C(G)$ we denote the set of all continuous functions on $G$, and by $C^{k, \beta}(\bar{D})$ (resp. $\left.C^{k, \beta}(D)\right)$ the space of functions whose $k$ th order partial derivatives are uniformly Hölder continuous (resp. locally Hölder continuous) with exponent $\beta$ in $D$. Furthermore, $\mathbb{L}_{p}$ (resp. $\mathbb{L}_{\infty}$ ) is the Banach space of measurable functions on $D$ that are $p$-integrable (resp. essentially bounded); $W_{2}^{1}$ is the usual Sobolev space of all elements $u$ of $\mathbb{L}_{2}$ having derivatives $D_{i} u$ in $\mathbb{L}_{2}$, and $\stackrel{\circ}{W}_{2}^{1}$ is the closure of the space of infinitely dif- 
ferentiable functions with compact supports lying in $D$ with respect to the $W_{2}^{1}$-norm $\|\cdot\|_{W_{2}^{1}}$. By $\|\cdot\|_{p}$ we denote the norm in $\mathbb{L}_{p} ;(\cdot, \cdot)_{2}$ denotes the scalar product in $\mathbb{L}_{2}$ whereas $(\cdot, \cdot)$ is the usual scalar product in $\mathbb{R}^{d} ;|D|$ is the Lebesgue measure of $D$. The abbreviation "a.e." means "almost everywhere with respect to the Lebesgue measure".

2. Uniqueness and a priori estimates. Let $\left\{\mathcal{F}_{t}\right\}_{t \geq 0}$ denote the minimum completed admissible filtration for $\mathbb{X}$.

Definition. Let $\varphi: \partial D \rightarrow \mathbb{R}$ and $f: D \times \mathbb{R} \times \mathbb{R}^{d} \rightarrow \mathbb{R}$ be measurable functions and let $x \in D$. We say that a pair $\left\{\left(Y_{t}^{x}, Z_{t}^{x}\right) ; t \geq 0\right\}$ of $\left\{\mathcal{F}_{t}\right\}_{t>0}$-progressively measurable processes is a solution of the $\operatorname{BSDE}(\varphi, f)$ associated with $\left(X, P_{x}\right)$ if $Y^{x}$ is continuous, $\int_{0}^{\tau}\left|Z_{t}^{x}\right|^{2} d t<\infty P_{x}$-a.s. and (1.6) is satisfied with the martingale additive functional $M$ of the decomposition (1.3).

In fact we will consider solutions with better integrability properties, namely solutions in the class $\mathcal{H}_{\alpha ; x}^{1+d}$ of all $\left\{\mathcal{F}_{t}\right\}_{t \geq 0}$-progressively measurable processes $\xi$ with values in $\mathbb{R}^{1+d}$ having a finite norm

$$
\|\xi\|_{\alpha ; x}^{2}=E_{x} \int_{0}^{\tau} e^{\alpha t}\left|\xi_{t}\right|^{2} d t
$$

for some $\alpha \geq 0$.

We will assume that $D$ is a bounded domain in $\mathbb{R}^{d}$ which is regular for $A$, that is, $P_{x}(\bar{\tau}=0)=1, x \in \partial D$, where $\bar{\tau}=\inf \left\{t>0:\left|X_{t}\right| \notin D\right\}$. We will need the following assumptions on $\varphi$ and $f$ :

(i) $\varphi: \partial D \rightarrow \mathbb{R}$ is continuous and can be extended by continuity to $\bar{D}$ so that the extended function (still denoted by $\varphi$ ) belongs to $W_{2}^{1}$;

(ii) $f: D \times \mathbb{R} \times \mathbb{R}^{d} \rightarrow \mathbb{R}$ satisfies the Carathéodory condition, that is, $f(x, \cdot, \cdot)$ is continuous for a.e. $x \in D$ and $f(\cdot, y, z)$ is measurable for each $(y, z) \in \mathbb{R} \times \mathbb{R}^{d}$

(iii) $\left(y_{1}-y_{2}\right) \cdot\left(f\left(x, y_{1}, z\right)-f\left(x, y_{2}, z\right)\right) \leq \mu\left|y_{1}-y_{2}\right|^{2}$ for some $\mu \in \mathbb{R}$ (the monotonicity condition);

(iv) $\left|f\left(x, y, z_{1}\right)-f\left(x, y, z_{2}\right)\right| \leq L\left|z_{1}-z_{2}\right|$ for some $L \geq 0$;

(v) $|f(x, y, 0)| \leq g(x)+K|y|$ for some $K \geq 0$ and non-negative $g \in \mathbb{L}_{p}$ with $p>2 \vee d$ if $2 \mu+L^{2} \leq 0$, and $g \in \mathbb{L}_{\infty}$ in the general case.

For $\mu, L$ we will assume that

(vi') $2 \mu+L^{2} \leq 0$ if $b \neq 0$ (non-symmetric case), or

$\left(\mathrm{vi}^{\prime \prime}\right) \sup _{a \in \mathcal{A}(\lambda, \Lambda)} \sup _{x \in D} E_{x}^{(a)} e^{\alpha \tau}<\infty$ for some $\alpha>2 \mu+L^{2}$ if $b=0$ (symmetric case). Here $E_{x}^{(a)}$ denotes expectation with respect to the measure $P_{x}^{(a)}$ corresponding to operator (1.1) with $b=0$ and $\mathcal{A}(\lambda, \Lambda)$ is the set of all measurable $a: D \rightarrow \mathbb{R}^{d} \otimes \mathbb{R}^{d}$ satisfying (1.2). 
REMARK 2.1. Since there exists $c=c(\lambda, \Lambda, d)$ such that

$$
p(t, x, y) \leq \frac{c}{t^{d / 2}} \exp \left(-\frac{|y-x|^{2}}{c t}\right)
$$

for all $t>0$ and $x, y \in \mathbb{R}^{d}$ (see, e.g., [16, Section I.1]), the proof of [3, Proposition 1.18] shows that there is $C>0$ depending only on $\lambda, \Lambda, d$ such that $\sup _{a \in \mathcal{A}(\lambda, \Lambda)} \sup _{x \in D} E_{x} e^{\alpha \tau}<\infty$ for any $\alpha<\alpha_{0} \equiv C|D|^{-2 / d}$. Therefore (vi') is satisfied if $2 \mu+L^{2}<\alpha_{0}$.

Proposition 2.2. If (i)-(iv) are satisfied and $E_{x} e^{\alpha \tau}<\infty$, where $\alpha=$ $2 \mu+L^{2}$, then the $\operatorname{BSDE}(\varphi, f)$ has at most one solution in the space $\mathcal{H}_{\alpha ; x}^{1+d}$.

Proof. See, e.g., the proof of [4, Proposition 3.2].

The remainder of this section is devoted to some a priori estimates on solutions of (1.6). These estimates will be needed in the next two sections.

Proposition 2.3. Assume (i)-(iv) and that $\|f(\cdot, 0,0)\|_{\alpha ; x}<\infty$ for some $\alpha \geq 0$. If $\left(Y^{x}, Z^{x}\right) \in \mathcal{H}_{0 ; x}^{1+d}$ is a solution of the $\operatorname{BSDE}(\varphi, f)$ such that

$$
E_{x} \sup _{0 \leq t \leq \tau} e^{\alpha t}\left|Y_{t}^{x}\right|^{2}<\infty
$$

then $\left(Y^{x}, Z^{x}\right) \in \mathcal{H}_{\alpha ; x}^{1+d}$ and for any $\delta, \varepsilon>0$,

$$
\begin{aligned}
E_{x}\left\{e^{\alpha(t \wedge \tau)}\left|Y_{t \wedge \tau}^{x}\right|^{2}\right. & \left.+\int_{t \wedge \tau}^{\tau} e^{\alpha s}\left(\left(\alpha-\alpha_{1}\right)\left|Y_{s}^{x}\right|^{2}+\varepsilon(1+\varepsilon)^{-1}\left|Z_{s}^{x}\right|^{2}\right) d s\right\} \\
& \leq E_{x}\left\{e^{\alpha \tau}\left|\varphi\left(X_{\tau}\right)\right|^{2}+\delta^{-1} \int_{t \wedge \tau}^{\tau} e^{\alpha s}\left|f\left(X_{s}, 0,0\right)\right|^{2} d s\right\}
\end{aligned}
$$

for all $t \geq 0$, where $\alpha_{1}=\delta+2 \mu+(1+\varepsilon) L^{2}$.

Proof. See, e.g., the proof of [4, Corollary 4.4.1].

For given $x \in D$ let $\mathcal{L}_{\alpha}(x)$ denote the space of functions having a finite norm

$$
\|u\|_{\alpha ; x}^{2}=E_{x} \int_{0}^{\tau} e^{\alpha t}\left|u\left(X_{t}\right)\right|^{2} d t=\|u(X)\|_{\alpha ; x}^{2}
$$

and let $\mathcal{W}_{\alpha}(x)$ denote the space of all elements of $\mathcal{L}_{\alpha}(x)$ having generalized derivatives $D_{i} u$ in $\mathcal{L}_{\alpha}(x)$ and equipped with the norm

$$
\|u\|_{\mathcal{W}_{\alpha}(x)}^{2}=\|u\|_{\alpha ; x}^{2}+\|\nabla u\|_{\alpha ; x}^{2} .
$$

Notice that by Fubini's theorem, $\|u\|_{\alpha ; x}^{2}=\int_{0}^{\infty} T_{t} u^{2}(x) d t=U_{\alpha} u^{2}(x)$, where $\left\{T_{t}, t \geq 0\right\}$ is the semigroup of positive linear operators on $\mathbb{L}_{\infty}$ defined by $T_{t} f(x)=E_{x} \mathbf{1}_{\{t<\tau\}} e^{\alpha t} f\left(X_{t}\right)$ and $U_{\alpha}$ is the potential operator for $\left\{T_{t}\right\}$. By (2.1) there is $C=C(\lambda, \Lambda, d)$ such that $T_{t} f(x) \leq C e^{\alpha t} P_{t}\left(\mathbf{1}_{D} f\right)(x)$, where $\left\{P_{t}, t \geq 0\right\}$ is the transition semigroup of a standard $d$-dimensional Wiener 
process. Hence, by [3, Theorem 3.10], for each $t>0, T_{t}$ is also a bounded operator from $\mathbb{L}_{1}$ to $\mathbb{L}_{\infty}$. Therefore, if $\sup _{x \in D} U_{\alpha} 1(x)<\infty$, then there is a measurable density $U_{\alpha}(\cdot, \cdot)$ such that

$$
\|u\|_{\alpha ; x}^{2}=\int_{D}|u(y)|^{2} U_{\alpha}(x, y) d y
$$

(see, e.g., the proof of Corollary to Theorem 3.18 in [3]). Thus $\mathcal{L}_{\alpha}(x)$ is in fact an $\mathbb{L}_{2}$-space with the weight $U_{\alpha}(x, \cdot)$. The function $U_{\alpha}$ will be called the $\alpha$-Green function for $A$ in $D$. In the case where $\alpha=0$ the $\alpha$-Green function reduces to the usual Green function which we will denote by $G_{D}$. Notice also that if $b=0$ then the operators $T_{t}$ are symmetric, and consequently $U_{\alpha}(x, y)=U_{\alpha}(y, x), x, y \in D$.

Suppose $u$ is a solution of (1.5) such that $(Y, Z)=\left(u\left(X^{\tau}\right),(\sigma \nabla u)\left(X^{\tau}\right)\right) \in$ $\mathcal{H}_{0, x}^{1+d}$ is a solution of (1.6) satisfying (2.2). Then from (2.3) with $t=0$ it follows that

$$
\begin{aligned}
|u(x)|^{2}+\left(\alpha-\alpha_{1}\right)\|u\|_{\alpha ; x}^{2}+ & \varepsilon(1+\varepsilon)^{-1}\|\sigma \nabla u\|_{\alpha ; x}^{2} \\
& \leq E_{x} e^{\alpha \tau}\left|\varphi\left(X_{\tau}\right)\right|^{2}+\delta^{-1}\|f(\cdot, 0,0)\|_{\alpha ; x}^{2} .
\end{aligned}
$$

Consider now the problem (1.5) with $f(x, y, z)=F(x)$, that is, the linear problem. Suppose $u$ is a solution of it such that (1.8) is a solution to (1.6) in the space $\mathcal{H}_{0, x}^{1+d}$. By Itô's formula, for any $n \in \mathbb{N}$ we have

$$
\begin{aligned}
&\left|Y_{0}^{x}\right|^{2}+\int_{0}^{\tau \wedge n}\left|Z_{s}^{x}\right|^{2} d s+2 \int_{0}^{\tau \wedge n}\left\langle Y_{s}^{x} Z_{s}^{x}, \sigma^{-1}\left(X_{s}\right) d M_{s}\right\rangle \\
&=\left|Y_{\tau \wedge n}^{x}\right|^{2}+2 \int_{0}^{\tau \wedge n} F\left(X_{s}\right) Y_{s}^{x} d s .
\end{aligned}
$$

Taking expectations and letting $n \rightarrow \infty$ gives

$$
E_{x}\left\{\left|Y_{0}^{x}\right|^{2}+\int_{0}^{\tau}\left|Z_{t}^{x}\right|^{2} d s\right\} \leq E_{x}\left\{\left|Y_{\tau}^{x}\right|^{2}+2 \int_{0}^{\tau} F\left(X_{t}\right) Y_{t}^{x} d t\right\},
$$

and so

$$
|u(x)|^{2}+\|\nabla u\|_{0 ; x}^{2} \leq E_{x}\left|\varphi\left(X_{\tau}\right)\right|^{2}+\|F\|_{0 ; x}^{2}+\|u\|_{0 ; x}^{2} .
$$

By (1.6),

$$
|u(x)| \leq E_{x}\left|\varphi\left(X_{\tau}\right)\right|+E_{x} \int_{0}^{\tau}\left|F\left(X_{t}\right)\right| d t
$$


Obviously $\|u\|_{0 ; x}^{2} \leq\|u\|_{\infty}^{2} E_{x} \tau$, so from (2.5), (2.6) we see that

$$
\begin{aligned}
\|\nabla u\|_{0 ; x}^{2} \leq & \left(1+2 E_{x} \tau\right)\|\varphi\|_{\infty}^{2}+\|F\|_{0 ; x}^{2} \\
& +2 E_{x} \tau \sup _{x \in D}\left(E_{x} \int_{0}^{\tau}\left|F\left(X_{t}\right)\right| d t\right)^{2} .
\end{aligned}
$$

3. Linear equations. It is well known that in case $d>1$ for any $\varphi \in W_{2}^{1}$ and $F \in \mathbb{L}_{2}$ the problem

$$
A u=-F,\left.\quad u\right|_{\partial D}=\varphi
$$

has a unique weak solution in $W_{2}^{1}$, that is, there exists a unique $u \in W_{2}^{1}$ such that $u-\varphi \in \stackrel{\circ}{W}_{2}^{1}$ and $(a \nabla u, \nabla \eta)_{2}-2(\nabla u, \eta b)_{2}=2(F, \eta)_{2}$ for all $\eta \in \stackrel{\circ}{W}_{2}^{1}$ (see, e.g., [5, Theorem 8.3]). If, in addition, $\varphi$ satisfies (i) and $F \in \mathbb{L}_{p}$ with $p>d / 2$ then $u$ is continuous on $\bar{D}([5$, Theorem 8.31$])$. In the case where $d=1$ the problem (3.1) has an even more regular weak solution for $F \in \mathbb{L}_{p}$ with $p=1$ (the substitution $v=a u^{\prime} / 2$ reduces $A u=-F$ to the ordinary linear differential equation $v^{\prime}+2 b a^{-1} v=-F$, so one can easily write down an explicit formula for the solution). Our purpose is to show that the condition $F \in \mathbb{L}_{p}$ can be replaced by

$$
\sup _{x \in D}\|F\|_{0 ; x}<\infty
$$

and, what is more important, that under (3.2) the pair $\left(u\left(X^{\tau}\right),(\sigma \nabla u)\left(X^{\tau}\right)\right)$ is a solution of the $\operatorname{BSDE}(\varphi, F)$. To prove this, we will need some auxiliary results.

Proposition 3.1. If $\varphi \in C(\partial D)$ and $F$ satisfies $(3.2)$ then $u: \bar{D} \rightarrow \mathbb{R}$ defined by

$$
u(x)=E_{x}\left\{\varphi\left(X_{\tau}\right)+\int_{0}^{\tau} F\left(X_{t}\right) d t\right\}, \quad x \in \bar{D}
$$

is continuous.

Proof. Since $D$ is regular, $P_{x}(\tau=\bar{\tau})=1$ for $x \in \bar{D}$. Hence, by standard arguments, $\bar{D} \ni x \mapsto E_{x} \varphi\left(X_{\tau}\right)$ is continuous (see, e.g., the proof of [3, Theorem 1.23]). Therefore we only need to show that if $\left\{x_{n}\right\} \subset \bar{D}$, $x \in \partial D$ and $x_{n} \rightarrow x$ then $E_{x_{n}} \int_{0}^{\tau} F\left(X_{t}\right) d t \rightarrow E_{x} \int_{0}^{\tau} F\left(X_{t}\right) d t=0$, and for this purpose it suffices to show that $E_{x_{n}} \tau \rightarrow 0$, because by Schwarz's inequality $\left|E_{x_{n}} \int_{0}^{\tau} F\left(X_{t}\right) d t\right| \leq\|F\|_{0, x}\left(E_{x_{n}} \tau\right)^{1 / 2}$. By [3, Proposition 1.19], for each $t>0$ the function $x \mapsto P_{x}(\tau>t)$ is upper semicontinuous in $\mathbb{R}^{d}$, so $\limsup _{n \rightarrow \infty} P_{x_{n}}(\tau>t) \leq P_{x}(\tau>t)=0$. On the other hand, since $P_{x}(\tau>t) \leq e^{-\alpha_{0} t / 2} \sup _{x \in D} E_{x} e^{\alpha_{0} \tau / 2}$ for $t \geq 0$, it follows from Remark 2.1 that the functions $t \mapsto P_{x}(\tau>t)$ are bounded by an integrable function 
uniformly in $x \in \bar{D}$. Therefore applying Fatou's lemma gives

$$
\limsup _{n \rightarrow \infty} E_{x_{n}} \tau=\limsup _{n \rightarrow \infty} \int_{0}^{\infty} P_{x_{n}}(\tau>t) d t \leq \int_{0}^{\infty} \limsup _{n \rightarrow \infty} P_{x_{n}}(\tau>t) d t=0,
$$

and the proof is complete.

Lemma 3.2. Let $x \in D$. If $u_{n} \rightarrow u$ in $\mathbb{L}_{2}$ and $\left\{u_{n}\right\}$ is bounded in $\mathcal{L}_{0}(x)$ then $u \in \mathcal{L}_{0}(x)$ and $\|u\|_{0 ; x} \leq \limsup _{n \rightarrow \infty}\left\|u_{n}\right\|_{0 ; x}$. If , in addition, $\left\{u_{n}\right\}$ is a Cauchy sequence in $\mathcal{L}_{0}(x)$ then $u_{n} \rightarrow u$ in $\mathcal{L}_{0}(x)$.

Proof. Let $\left\{V_{m}\right\}$ be a sequence of open sets such that $x \notin \bar{V}_{m} \subset V_{m+1} \subset$ $\bar{V}_{m+1} \subset D$ for $m \in \mathbb{N}$ and $\bigcup_{m=1}^{\infty} V_{m}=D$. It is known that $G_{D}(x, \cdot)$ is continuous on $D \backslash\{x\}$ (see [14]). Therefore $G_{D}(x, \cdot)$ is bounded on $V_{m}$, and consequently $\left\|\mathbf{1}_{V_{m}}\left(u_{n}-u\right)\right\|_{0 ; x}^{2}=\int_{V_{m}}\left|u_{n}(y)-u(y)\right|^{2} G_{D}(x, y) d y \rightarrow 0$, that is, $\mathbf{1}_{V_{m}} u_{n} \rightarrow \mathbf{1}_{V_{m}} u$ in $\mathcal{L}_{0}(x)$. On the other hand, $\left\{u_{n}\right\}$ is bounded in $\mathcal{L}_{0}(x)$ and therefore weakly relatively compact. Suppose $u_{n(k)} \rightarrow v$ weakly in $\mathcal{L}_{0}(x)$ for some subsequence $\{n(k)\}$. Then $\mathbf{1}_{V_{m}} u_{n(k)} \rightarrow \mathbf{1}_{V_{m}} v$ weakly in $\mathcal{L}_{0}(x)$ as well. It follows that $\left\|\mathbf{1}_{V_{m}} u\right\|_{0 ; x}=\left\|\mathbf{1}_{V_{m}} v\right\|_{0 ; x}$. Since $\mathbf{1}_{V_{m}} u^{2} \uparrow \mathbf{1}_{D \backslash\{x\}} u^{2}$ and $\mathbf{1}_{V_{m}} v^{2} \uparrow \mathbf{1}_{D \backslash\{x\}} v^{2}$ pointwise, using the monotone convergence theorem yields $\|u\|_{0 ; x}=\|v\|_{0, x}$. Let $(\cdot, \cdot)_{0 ; x}$ denote the scalar product in $\mathcal{L}_{0}(x)$. Since $\left(u_{n(k)}, v\right)_{0 ; x} \rightarrow(v, v)_{0 ; x}$, it follows that $\left|\left(u_{n(k)}, v\right)_{0 ; x}\right| \rightarrow(v, v)_{0 ; x}$, hence

$$
\begin{aligned}
\|v\|_{0 ; x}^{2}=\limsup _{k \rightarrow \infty}\left|\left(u_{n(k)}, v\right)_{0 ; x}\right| & \leq \limsup _{k \rightarrow \infty}\left\|u_{n(k)}\right\|_{0 ; x} \cdot\|v\|_{0 ; x} \\
& \leq \limsup _{n \rightarrow \infty}\left\|u_{n}\right\|_{0 ; x} \cdot\|v\|_{0 ; x}
\end{aligned}
$$

which proves the first assertion. The second one follows from the fact that if $u_{n} \rightarrow v$ in $\mathcal{L}_{0}(x)$ then $\left\|\mathbf{1}_{V_{m}}(u-v)\right\|_{0 ; x}=0$ for $m \in \mathbb{N}$.

For $n \in \mathbb{N}$ let $a_{n}: \mathbb{R}^{d} \rightarrow \mathbb{R}^{d} \otimes \mathbb{R}^{d}$ and $b_{n}: \mathbb{R}^{d} \rightarrow \mathbb{R}^{d}$ be measurable functions satisfying (1.2) and let $\varphi_{n} \in W_{2}^{1}$ and $F_{n} \in \mathbb{L}_{2}$. Set

$$
A^{n}=\frac{1}{2} \sum_{i, j=1}^{d} D_{j}\left(a_{n}^{i j}(x) D_{i}\right)+\sum_{i=1}^{d} b_{n}^{i}(x) D_{i}
$$

and let $u_{n} \in W_{2}^{1}$ be a weak solution of the problem

$$
A^{n} u_{n}=-F_{n},\left.\quad u_{n}\right|_{\partial D}=\varphi_{n} .
$$

In the rest of this section we will assume that $a_{n}^{i j} \rightarrow a^{i j}$ and $b_{n}^{i} \rightarrow b^{i}$ a.e. for $i, j=1, \ldots, d$.

The following proposition is undoubtedly known. We provide a proof, because we could not find an appropriate reference.

Proposition 3.3. If $\varphi_{n} \rightarrow \varphi$ in $W_{2}^{1}$ and $F_{n} \rightarrow F$ in $\mathbb{L}_{2}$ then $u_{n} \rightarrow u$ in $W_{2}^{1}$, where $u$ is a unique weak solution of the problem (3.1). 
Proof. First we show that there is $C=C(\lambda, \Lambda)$ such that

$$
\|u\|_{2} \leq C\|F\|_{2}
$$

for some $C=C(\lambda, \Lambda)$. We can certainly assume that $F$ is bounded, for if $\left\{f_{k}\right\}$ is a sequence of bounded functions such that $f_{k} \rightarrow F$ in $\mathbb{L}_{2}$ and $u_{k}$ is a weak solution of (3.1) with $F$ replaced by $f_{k}, k \in \mathbb{N}$, then $\|u\|_{2}=\lim _{k \rightarrow \infty}\left\|u_{k}\right\|_{2}$ by [5, Corollary 8.7]. Let $\widehat{A}=\sum_{i, j=1}^{d} D_{j}\left(\frac{1}{2} a^{i j}(x) D_{i}-b^{j}(x)\right)$ be the formal adjoint of $A$. By [5, Theorem 8.6] there exists a unique weak solution $v \in$ $\stackrel{\circ}{W}_{2}^{1}$ of the problem $\widehat{A} v=-1,\left.v\right|_{\partial D}=0$. Since, by [5, Theorem 8.15], $u, v$ are bounded, it follows that $u v \in \stackrel{\circ}{W}_{2}^{1}$, and hence $-2^{-1}(a \nabla u, \nabla(u v))_{2}+$ $(\nabla u, u v b)_{2}=-(F, u v)_{2}$. Therefore

$$
(a \nabla u, v \nabla u)_{2}+(a \nabla u, u \nabla v)_{2}-2(\nabla u, u v b)_{2}=2(F, u v)_{2} .
$$

On the other hand, $-2^{-1}\left(a \nabla v, \nabla u^{2}\right)_{2}+\left(v b, \nabla u^{2}\right)_{2}=-\left(u^{2}, 1\right)_{2}$, and consequently

$$
\|u\|_{2}^{2}=(a \nabla u, u \nabla v)_{2}-2(\nabla u, u v b)_{2} .
$$

By the above,

$$
\|u\|_{2}^{2}+(a \nabla u, v \nabla u)_{2}=2(F, u v)_{2},
$$

and so $\|u\|_{2} \leq\|v\|_{\infty}\|F\|_{2}$, which proves (3.5), because from [5, Theorem 8.16] it follows that $v$ is bounded by a constant depending only on $\lambda, \Lambda$.

Now, write $w_{n}=u_{n}-\varphi_{n}-u+\varphi$. Since $w_{n} \in \stackrel{\circ}{W}_{2}^{1}$,

$$
\begin{aligned}
\lambda\left\|\nabla w_{n}\right\|_{2}^{2} \leq & \left(a_{n} \nabla w_{n}, \nabla w_{n}\right)_{2} \\
= & \left(a_{n} \nabla u_{n}, \nabla w_{n}\right)_{2}-\left(a_{n} \nabla \varphi_{n}, \nabla w_{n}\right)_{2} \\
& +\left(\left(a-a_{n}\right) \nabla(u-\varphi), \nabla w_{n}\right)_{2}-\left(a \nabla u, \nabla w_{n}\right)_{2}+\left(a \nabla \varphi, \nabla w_{n}\right)_{2} \\
= & 2\left(F_{n}-F, w_{n}\right)_{2}+\left(\left(a-a_{n}\right) \nabla u, \nabla w_{n}\right)_{2}+\left(a_{n} \nabla\left(\varphi-\varphi_{n}\right), \nabla w_{n}\right)_{2} \\
& +2\left(\nabla w_{n}, w_{n} b_{n}\right)_{2}+2\left(\nabla\left(\varphi_{n}-\varphi\right), w_{n} b\right)_{2}+2\left(\nabla u, w_{n}\left(b_{n}-b\right)\right)_{2} .
\end{aligned}
$$

From this and (3.5) the proposition follows.

Let $\mathbb{X}^{n}=\left\{\left(X, P_{x}^{n}\right) ; x \in \mathbb{R}^{d}\right\}$ be a Markov process corresponding to the operator $A^{n}$ and let $M^{n}$ denote the martingale additive functional of $\mathbb{X}^{n}$ of the decomposition of $X$ of the form (1.3).

Lemma 3.4. $\mathcal{L}\left[\left(X, M^{n}\right) \mid P_{x}^{n}\right] \Rightarrow \mathcal{L}\left[(X, M) \mid P_{x}\right]$ in $C\left([0, \infty) ; \mathbb{R}^{2 d}\right)$ for each $x \in D$.

Proof. Since $P_{x}^{n} \Rightarrow P_{x}$ in $C\left([0, \infty) ; \mathbb{R}^{d}\right.$ ) (see, e.g., [16]), it suffices to repeat, with some obvious changes, the proof of [11, Lemma 3.2].

In the proof of the following lemma we combine ideas from the proofs of [3, Theorem A.1] and [17, Lemma 11.1.2]. 
Lemma 3.5. Let $x \in D$. There exists a sequence of regular domains $\left\{D_{m}\right\}$ such that $\{x\} \subset D_{m} \subset \bar{D}_{m} \subset D$ for $m \in \mathbb{N}, \bigcup_{m=1}^{\infty} D_{m}=D$ and $\tau_{m}=\inf \left\{t \geq 0:\left|X_{t}\right| \notin D_{m}\right\}$ is continuous at $\omega$ for $P_{x}$-almost all $\omega \in \Omega$.

Proof. Set $\varepsilon_{0}=\operatorname{dist}(x, \partial D)$. For $\varepsilon \in\left(0, \varepsilon_{0}\right)$ let $D_{\varepsilon}=\{y \in D: \operatorname{dist}(y, \partial D)$ $>\varepsilon\}$ and let $E_{\varepsilon}$ denote the connected component of $D_{\varepsilon}$ containing $x$. In the proof of [3, Theorem A.1] it is shown that the sets $E_{\varepsilon}$ are regular and $\bigcup_{0<\varepsilon<\varepsilon_{0}} E_{\varepsilon}=D$, so it is clear that the lemma will be proved once we show that except for at most countably many $\varepsilon$ 's from $\left(0, \varepsilon_{0}\right), \sigma_{\varepsilon}=\inf \{t \geq 0$ : $\left.\left|X_{t}\right| \notin E_{\varepsilon}\right\}$ is continuous for $P_{x}$-almost all $\omega$ in $\Omega$. Let $\sigma_{\varepsilon}^{+}=\inf \{t \geq 0$ : $\left.\left|X_{t}\right| \notin \bar{E}_{\varepsilon}\right\}$. Since $\sigma_{\varepsilon}^{+}=\lim _{\delta \downarrow \varepsilon} \sigma_{\delta}$ and $\varepsilon \mapsto E_{x} \exp \left(-\sigma_{\varepsilon}\right)$ is a non-negative non-increasing function,

$$
E_{x} \exp \left(-\sigma_{\varepsilon}^{+}\right)=\lim _{\delta \downarrow \varepsilon} E_{x} \exp \left(-\sigma_{\delta}\right)=E_{x} \exp \left(-\sigma_{\varepsilon}\right)
$$

for all but a countable number of $\varepsilon$ 's. Since $\sigma_{\varepsilon}^{+} \geq \sigma_{\varepsilon}$ this shows that $\sigma_{\varepsilon}^{+}=\sigma_{\varepsilon}$ for all but a countable number of $\varepsilon$ 's, which is the desired conclusion because $\sigma_{\varepsilon}$ is lower semicontinuous and $\sigma_{\varepsilon}^{+}$is upper semicontinuous.

Lemma 3.6. For given $x \in D$ let $\left\{D_{m}\right\}$ be the sequence of domains of Lemma 3.5 and let $\left\{\tau_{m}\right\}$ be the sequence of the corresponding stopping times. Assume $F_{n}, F: D \rightarrow \mathbb{R}$ and $G_{n}=\left(G_{n}^{1}, \ldots, G_{n}^{d}\right), G=\left(G^{1}, \ldots, G^{d}\right): \mathbb{R}^{d} \rightarrow \mathbb{R}$ are measurable functions such that $F_{n} \rightarrow F$ and $G_{n}^{i} \rightarrow G^{i}, i=1, \ldots, d$, in $\mathbb{L}_{2}$. Then for any $m, N \in \mathbb{N}$ and $\delta>0$,

$$
\begin{aligned}
\mathcal{L} & {\left[\left(X_{\left(\cdot \wedge N \wedge \tau_{m}\right) \vee \delta}, \quad \int_{\delta}^{\left(\cdot \wedge N \wedge \tau_{m}\right) \vee \delta} F_{n}\left(X_{t}\right) d t, \quad \int_{\delta}^{\left(\cdot \wedge N \wedge \tau_{m}\right) \vee \delta}\left\langle G_{n}\left(X_{t}\right), d M_{t}^{n}\right\rangle\right) \mid P_{x}^{n}\right] } \\
& \Rightarrow \mathcal{L}\left[\left(X_{\left(\cdot \wedge N \wedge \tau_{m}\right) \vee \delta}, \quad \int_{\delta}^{\left(\cdot \wedge N \wedge \tau_{m}\right) \vee \delta} F\left(X_{t}\right) d t, \int_{\delta}^{\left(\cdot \wedge N \wedge \tau_{m}\right) \vee \delta}\left\langle G\left(X_{t}\right), d M_{t}\right\rangle\right) \mid P_{x}\right]
\end{aligned}
$$

in $C\left([0, \infty) ; \mathbb{R}^{d+2}\right)$ as $n \rightarrow \infty$.

Proof. By Lemmas 3.4 and 3.5,

$$
\begin{aligned}
\mathcal{L}\left[\left(\left(N \wedge \tau_{m}\right) \vee \delta, X_{\left(\cdot \wedge N \wedge \tau_{m}\right)} \vee \delta\right.\right. & \left.\left.M^{n}\right) \mid P_{x}^{n}\right] \\
& \Rightarrow \mathcal{L}\left[\left(\left(N \wedge \tau_{m}\right) \vee \delta, X_{\left(\cdot \wedge N \wedge \tau_{m}\right) \vee \delta}, M\right) \mid P_{x}\right]
\end{aligned}
$$

in $\mathbb{R} \times C\left([0, \infty) ; \mathbb{R}^{2 d}\right)$ as $n \rightarrow \infty$. On the other hand, by (2.1), for any $h \in \mathbb{L}_{1}$,

$$
\begin{aligned}
E_{x}^{n} \int_{\delta}^{\left(N \wedge \tau_{m}\right) \vee \delta}\left|h\left(X_{t}\right)\right| d t & \leq C(\lambda, \Lambda) \int_{\delta}^{N \vee \delta} t^{-d / 2} d t \int_{D_{m}}|h(y)| d y \\
& \leq C(\lambda, \Lambda, d, N, \delta)\|h\|_{1},
\end{aligned}
$$


and similar estimates hold if we replace $E_{x}^{n}$ by $E_{x}$. Therefore the lemma follows by the same method as in the proof of [12, Theorem 1].

We are now ready to prove our main result on the Dirichlet problem for linear equations.

THEOREM 3.7. If $\varphi$ satisfies (i) and $F \in \mathbb{L}_{2}$ satisfies (3.2) then there exists a unique weak solution $u \in W_{2}^{1} \cap C(\bar{D})$ of the problem (3.1). Moreover, for each $x \in D$ the pair (1.8) is a solution, in $\mathcal{H}_{0 ; x}^{1+d}$, of the $\operatorname{BSDE}(\varphi, F)$ associated with $\left(X, P_{x}\right)$.

Proof. We first prove the theorem under the additional assumption that $F$ is bounded. Let $j(x)=c \exp \left(-1 /\left(1-|x|^{2}\right)\right)$ if $|x|<1$ and $j(x)=0$ if $|x| \geq 1$, where $c$ is chosen so that $\int_{\mathbb{R}^{d}} j(x) d x=1$, and let $j_{n}(x)=n^{d} j(n x)$. Set

$$
a_{n}^{i j}=a^{i j} * j_{n}, \quad b_{n}^{i}=b^{i} * j_{n}, \quad \varphi_{n}=\varphi * j_{n}, \quad F_{n}=F * j_{n}
$$

(we first extend $\varphi$ to a continuous function on $\mathbb{R}^{d}$ and $F$ to be zero outside $D$ ) and let $u_{n} \in C^{2}(D) \cap C(\bar{D})$ be a (unique) solution to the problem (3.4). Then for any $n, m, N \in \mathbb{N}$,

$$
\begin{aligned}
u_{n}\left(X_{t \wedge N \wedge \tau_{m}}\right)= & u_{n}\left(X_{N \wedge \tau_{m}}\right)+\int_{t \wedge N \wedge \tau_{m}}^{N \wedge \tau_{m}} F_{n}\left(X_{s}\right) d s \\
& -\int_{t \wedge N \wedge \tau_{m}}^{N \wedge \tau_{m}}\left\langle\nabla u_{n}\left(X_{s}\right), d M_{s}^{n}\right\rangle, \quad t \geq 0, P_{x}^{n} \text {-a.s. }
\end{aligned}
$$

By Proposition 3.3, $u_{n} \rightarrow u$ in $W_{2}^{1}$, where $u \in W_{2}^{1}$ is a unique solution of the problem (3.1). On the other hand, by (2.6), $\left\{u_{n}\right\}$ is bounded on $D$ and hence, by De Giorgi's continuity theorem (see [5, Theorem 8.24]), equicontinuous in each compact subset of $D$. Therefore there is a bounded continuous (on $D$ ) version of $u$ (still denoted by $u$ ) such that $u_{n} \rightarrow u$ uniformly on compact subsets of $D$. As a consequence, by Lemma 3.6 and the continuous mapping theorem (see [2, Theorem 5.5]),

$$
\begin{gathered}
\mathcal{L}\left[\left(u_{n}\left(X_{\left(\cdot \wedge N \wedge \tau_{m}\right) \vee \delta}\right), \quad \int_{\delta}^{\left(\cdot \wedge N \wedge \tau_{m}\right) \vee \delta} F_{n}\left(X_{t}\right) d t, \int_{\delta}^{\left(\cdot \wedge N \wedge \tau_{m}\right) \vee \delta}\left\langle\nabla u_{n}\left(X_{t}\right), d M_{t}^{n}\right\rangle\right) \mid P_{x}^{n}\right] \\
\Rightarrow \mathcal{L}\left[\left(u\left(X_{\left(\cdot \wedge N \wedge \tau_{m}\right) \vee \delta}\right), \int_{\delta}^{\left(\cdot \wedge N \wedge \tau_{m}\right) \vee \delta} F\left(X_{t}\right) d t, \int_{\delta}^{\left(\cdot \wedge N \wedge \tau_{m}\right) \vee \delta}\left\langle\nabla u\left(X_{t}\right), d M_{t}\right\rangle\right) \mid P_{x}\right]
\end{gathered}
$$

in $C\left([0, \infty) ; \mathbb{R}^{3}\right)$. By the above and (3.8), using once again the continuous mapping theorem, we get 


$$
\begin{aligned}
u\left(X_{\left(t \wedge N \wedge \tau_{m}\right) \vee \delta}\right)= & u\left(X_{\left(N \wedge \tau_{m}\right) \vee \delta}\right)+\int_{\left(t \wedge N \wedge \tau_{m}\right) \vee \delta}^{\left(N \wedge \tau_{m}\right) \vee \delta} F\left(X_{s}\right) d s \\
& -\int_{\left(t \wedge N \wedge \tau_{m}\right) \vee \delta}^{\left(N \wedge \tau_{m}\right) \vee \delta}\left\langle\nabla u\left(X_{s}\right), d M_{s}\right\rangle, \quad t \geq 0, P_{x^{-}} \text {a.s. }
\end{aligned}
$$

Letting $\delta \downarrow 0$ and then $N \uparrow \infty$ we see that

$$
u\left(X_{t \wedge \tau_{m}}\right)=u\left(X_{\tau_{m}}\right)+\int_{t \wedge \tau_{m}}^{\tau_{m}} F\left(X_{s}\right) d s-\int_{t \wedge \tau_{m}}^{\tau_{m}}\left\langle\nabla u\left(X_{s}\right), d M_{s}\right\rangle \quad P_{x^{-}} \text {a.s. }
$$

for $t \geq 0$. Since $\tau_{m} \uparrow \tau P_{x}$-a.s. and $F$ is bounded, $\int_{t \wedge \tau_{m}}^{\tau_{m}} F\left(X_{s}\right) d s \rightarrow$ $\int_{t \wedge \tau}^{\tau} F\left(X_{s}\right) d s P_{x}$-a.s. Furthermore, by (2.5) and boundedness of $\left\{u_{n}\right\},\left\{\nabla u_{n}\right\}$ is bounded in $\mathcal{L}_{0}(x)$ for each $x \in D$. From this and Lemma 3.2 we conclude that $\nabla u \in \mathcal{L}_{0}(x)$ for $x \in D$, hence that $\int_{t \wedge \tau_{m}}^{\tau_{m}}\left\langle\nabla u\left(X_{s}\right), d M_{s}\right\rangle \rightarrow$ $\int_{t \wedge \tau}^{\tau}\left\langle\nabla u\left(X_{s}\right), d M_{s}\right\rangle P_{x}$-a.s. Finally, by Proposition 3.1, $u$ is continuous in $\bar{D}$ and $u=\varphi$ on $\partial D$. Hence $u\left(X_{t \wedge \tau_{m}}\right) \rightarrow u\left(X_{t \wedge \tau}\right)$ and $u\left(X_{\tau_{m}}\right) \rightarrow \varphi\left(X_{\tau}\right)$ $P_{x}$-a.s. Thus, for each $x \in D$,

$$
u\left(X_{t \wedge \tau}\right)=\varphi\left(X_{\tau}\right)+\int_{t \wedge \tau}^{\tau} F\left(X_{s}\right) d s-\int_{t \wedge \tau}^{\tau}\left\langle\nabla u\left(X_{s}\right), d M_{s}\right\rangle \quad P_{x^{-}} \text {a.s. }
$$

for each $t \geq 0$. Since the processes on the left and right-hand side of the above equality are continuous, this proves the proposition for bounded $F$.

To prove the general case, we set $F_{n}=(-n) \vee F \wedge n$. By what has already been proved, the pair $\left(u_{n}\left(X^{\tau}\right),\left(\sigma \nabla u_{n}\right)\left(X^{\tau}\right)\right)$, where $u_{n} \in W_{2}^{1} \cap C(\bar{D})$ is a solution of the problem $A u_{n}=-F_{n},\left.u_{n}\right|_{\partial D}=\varphi$, solves the $\operatorname{BSDE}\left(\varphi, F_{n}\right)$. In particular,

$$
\begin{aligned}
u_{n}\left(X_{t \wedge \tau_{m}}\right)= & \varphi\left(X_{\tau}\right)+\int_{t \wedge \tau_{m}}^{\tau} F_{n}\left(X_{s}\right) d s \\
& -\int_{t \wedge \tau_{m}}^{\tau}\left\langle\nabla u_{n}\left(X_{s}\right), d M_{s}\right\rangle, \quad t \geq 0, P_{x^{-}} \text {a.s. }
\end{aligned}
$$

Clearly $\left|F_{n}-F\right| \rightarrow 0$ and $\left|F_{n}-F\right| \leq F$. Therefore, since $F$ satisfies (3.2), the Lebesgue dominated convergence theorem shows that

$$
\lim _{n \rightarrow \infty}\left\|F_{n}-F\right\|_{0 ; x}^{2}=\lim _{n \rightarrow \infty} \int_{D}\left|F_{n}-F\right|^{2}(y) G_{D}(x, y) d y=0
$$

for $x \in D$. Similarly, since $F \in \mathbb{L}_{2}$, we have $F_{n} \rightarrow F$ in $\mathbb{L}_{2}$, and consequently, by Proposition 3.3, $u_{n} \rightarrow u$ in $W_{2}^{1}$, where $u$ is a unique solution of (3.1). Moreover, for each $x \in D$,

$$
\nabla u_{n} \rightarrow \nabla u \quad \text { in } \mathcal{L}_{0}(x) .
$$


Indeed, from (2.7) it follows that for any $n, m \in \mathbb{N}$,

$$
\left\|\nabla\left(u_{n}-u_{m}\right)\right\|_{0 ; x}^{2} \leq\left\|F_{n}-F_{m}\right\|_{0 ; x}^{2}+2\left(E_{x} \tau\right) \sup _{x \in D}\left(\int_{0}^{\tau}\left|F_{n}-F_{m}\right|\left(X_{t}\right) d t\right)^{2} .
$$

By (3.10), $\lim _{n, m \rightarrow \infty}\left\|F_{n}-F_{m}\right\|_{0 ; x}^{2}=0$. At the same time, since $\left|F_{n}-F_{m}\right| \leq$ $|F|$ and $\left|F_{n}-F_{m}\right|=0$ if $|F| \leq N$ and $n, m \geq N$, for any $n, m \geq N$ we have

$$
\begin{aligned}
\sup _{x \in D} E_{x} \int_{0}^{\tau} \mid F_{n} & -F_{m}\left|\left(X_{t}\right) d t \leq \sup _{x \in D} E_{x} \int_{0}^{\tau} \mathbf{1}_{\{|F|>N\}}\right| F \mid\left(X_{t}\right) d t \\
& \leq \sup _{x \in D}\left(E_{x} \int_{0}^{\tau} \mathbf{1}_{\{|F|>N\}}\left(X_{t}\right) d t \cdot E_{x} \int_{0}^{\tau}\left|F\left(X_{t}\right)\right|^{2} d t\right)^{1 / 2} \\
& \leq N^{-1} \sup _{x \in D}\|F\|_{0 ; x}^{2} .
\end{aligned}
$$

Therefore $\left\{\nabla u_{n}\right\}$ is a Cauchy sequence in $\mathcal{L}_{0}(x)$. On the other hand, for any $V_{m}$ defined in the proof of Lemma 3.2 we have

$$
\begin{aligned}
\limsup _{n \rightarrow \infty}\left\|\mathbf{1}_{V_{m}} \nabla\left(u_{n}-u\right)\right\|_{0 ; x}^{2} & \\
& \leq \sup _{y \in V_{m}} G_{D}(x, y) \cdot \limsup _{n \rightarrow \infty} \int_{V_{m}}\left|\nabla\left(u_{n}-u\right)\right|^{2}(y) d y=0,
\end{aligned}
$$

so (3.11) follows. We now observe that by De Giorgi's continuity theorem (see [5, Theorem 8.24]), for any compact subset $K$ of $D$ and any $x, y \in K$ and $N \in \mathbb{N}$ we have

$$
\sup _{n \leq N}\left|u_{n}(x)-u_{n}(y)\right| \leq C_{1} \sup _{n \leq N}\left(\left\|u_{n}\right\|_{2}+\lambda^{-1}\left\|F_{n}\right\|_{d}\right)|x-y|^{\alpha}
$$

where $C_{1}=C_{1}(\lambda, \Lambda, d, \operatorname{dist}(K, \partial D))$ and $\alpha=\alpha(\lambda, \Lambda, d)>0$. Moreover,

$$
\begin{aligned}
\sup _{n>N} \mid & u_{n}(x)-u_{n}(y) \mid \\
& \leq\left|u_{N}(x)-u_{N}(y)\right|+\sup _{n>N}\left(\left|u_{n}(x)-u_{N}(x)\right|+\left|u_{n}(y)-u_{N}(y)\right|\right) \\
& \leq\left|u_{N}(x)-u_{N}(y)\right|+2 \sup _{n>N} \sup _{x \in K} E_{x} \int_{0}^{\tau}\left|F_{n}-F_{N}\right|\left(X_{t}\right) d t .
\end{aligned}
$$

Analysis similar to that in the proof of (3.12) shows that the second term on the right-hand side of the last inequality is bounded by $2 N^{-1} \sup _{x \in K}\|F\|_{0 ; x}^{2}$. Since $\left\|u_{n}\right\|_{2} \leq C_{2}\left(\|\varphi\|_{W_{2}^{1}}+\|F\|_{2}\right)$ for some $C_{2}$ not depending on $n$ (see [5, Corollary 8.7] or the proof of Proposition 3.3), putting together the above 
estimates yields

$$
\begin{aligned}
\sup _{n \in \mathbb{N}}\left|u_{n}(x)-u_{n}(y)\right| \leq & 2 C_{1}\left(C_{2}\left(\|\varphi\|_{W_{2}^{1}}+\|F\|_{2}\right)+\lambda^{-1} N|D|^{d}\right)|x-y|^{\alpha} \\
& +2 N^{-1} \sup _{x \in K}\|F\|_{0 ; x}^{2} .
\end{aligned}
$$

This proves that $\left\{u_{n}\right\}$ is equicontinuous in $K$. By (2.6) and (3.2) it is also bounded on any compact subset of $D$, and hence, by the Ascoli-Arzelà theorem, converges to $u$ uniformly on any such subset. Using this and (3.10), (3.11), and letting $n \rightarrow \infty$ in (3.9) gives

$$
u\left(X_{t \wedge \tau_{m}}\right)=\varphi\left(X_{\tau}\right)+\int_{t \wedge \tau_{m}}^{\tau} F\left(X_{s}\right) d s-\int_{t \wedge \tau_{m}}^{\tau}\left\langle\nabla u\left(X_{t}\right), d M_{s}\right\rangle, \quad t \geq 0,
$$

$P_{x^{-}}$a.s. Hence, letting $m \rightarrow \infty$ we obtain (3.9), because $\tau_{m} \uparrow \tau P_{x^{-a}}$.s. and $u$ is continuous on $\bar{D}$. This proves the theorem.

4. Semilinear PDEs and BSDEs. In this section we prove our main theorem on connections between weak solutions of the problem (1.5) and solutions of BSDEs of the form (1.6). We begin with results on uniqueness, existence and regularity of solutions of (1.5).

Before proving the first theorem we observe that if $\sup _{x} E_{x} e^{\alpha \tau}<\infty$ then $U_{\alpha} 1$ is a bounded weak solution of the problem $(\alpha+A) U_{\alpha} 1=-1$, $\left.U_{\alpha} 1\right|_{\partial D}=0$. This follows from the fact that for bounded $f$ the function $G_{D} f$ is a weak solution of the problem $A G_{D} f=-f,\left.G_{D} f\right|_{\partial D}=0$ and $G_{D}\left(\alpha U_{\alpha} 1\right)=U_{\alpha} 1-G_{D} 1$, because using the Markov property and integration by parts gives

$$
\begin{aligned}
E_{x} \int_{0}^{\tau} \alpha\left(E_{X_{t}} \int_{0}^{\tau} e^{\alpha s} d s\right) d t & =E_{x} \int_{0}^{\tau} E_{x}\left(\left(e^{\alpha \tau}-1\right) \mid \mathcal{F}_{t}\right) \alpha e^{-\alpha t} d t \\
& =E_{x}\left(e^{\alpha \tau}-1\right) .
\end{aligned}
$$

THEOREM 4.1. Assume that $\varphi \in W_{2}^{1}$ and $f$ satisfies (ii)-(v). If either $\mu+L^{2} \leq 0$, or $b=0$ and $\sup _{x \in D} E_{x} e^{\alpha \tau}<\infty$ for some $\alpha \geq 2 \mu+L^{2}$, then the problem (1.5) cannot have more than one weak solution in $W_{2}^{1}$.

Proof. Suppose that $u_{1}, u_{2} \in W_{2}^{1}$ are weak solutions of (1.5). Then $u=$ $u_{1}-u_{2} \in \dot{W}_{2}^{1}$ and $u$ solves the problem (3.1) with $F=F_{u_{1}}-F_{u_{2}}, \varphi=0$. Let $\left\{F_{n}\right\}$ be a sequence of bounded measurable functions such that $F_{n} \rightarrow F$ in $\mathbb{L}_{2}$, and for $n \in \mathbb{N}$ let $w_{n} \in \mathscr{W}_{2}^{1}$ be a weak solution of the problem $A w_{n}=-F_{n},\left.w_{n}\right|_{\partial D}=0$. Assume that $b=0$. As in the proof of (3.6) one can show that

$$
\left\|w_{n}\right\|_{2}^{2}+\alpha\left(w_{n}, w_{n} U_{\alpha} 1\right)_{2}+\left(a \nabla w_{n}, U_{\alpha} 1 \nabla w_{n}\right)_{2}=2\left(F_{n}, w_{n} U_{\alpha} 1\right)_{2} .
$$


By Proposition $3.3, w_{n} \rightarrow u$ in $W_{2}^{1}$, so letting $n \rightarrow \infty$ we obtain

$$
\|u\|_{2}^{2}+\alpha\left(u, u U_{\alpha} 1\right)_{2}+\left(a \nabla u, U_{\alpha} 1 \nabla u\right)_{2}=2\left(F, u U_{\alpha} 1\right)_{2} .
$$

On the other hand, by (iii), (iv),

$$
\begin{aligned}
2\left(F, u U_{\alpha} 1\right)_{2} & \leq 2 \mu\left(u, u U_{\alpha} 1\right)_{2}+2 L\left(|u| \cdot|\sigma \nabla u|, U_{\alpha} 1\right)_{2} \\
& \leq 2 \mu\left(u, u U_{\alpha} 1\right)_{2}+\left(a \nabla u, U_{\alpha} 1 \nabla u\right)_{2}+L^{2}\left(u, u U_{\alpha} 1\right)_{2} .
\end{aligned}
$$

Hence $\|u\|_{2}^{2}+\alpha\left(u, u U_{\alpha} 1\right)_{2} \leq\left(2 \mu+L^{2}\right)\left(u, u U_{\alpha} 1\right)_{2}$, and the theorem follows.

Consider now the case $b \neq 0$. Let $\widehat{A}$ be the formal adjoint of $A$ and let $\widehat{G}_{D} 1(x)=\int_{D} G(y, x) d y, x \in D$. Since $\widehat{G}_{D} 1 \in \stackrel{\circ}{W}_{2}^{1}$ is a weak solution of the problem $\widehat{A} \widehat{G}_{D} 1=-1,\left.\widehat{G}_{D} 1\right|_{\partial D}=0$, analysis similar to that in the proof of (4.1) shows that

$$
\|u\|_{2}^{2}+\left(a \nabla u, \widehat{G}_{D} 1 \nabla u\right)_{2}=2\left(F, u \widehat{G}_{D} 1\right)_{2} .
$$

Hence $\|u\|_{2}^{2} \leq\left(2 \mu+L^{2}\right)\left(u, u \widehat{G}_{D} 1\right)_{2}$, and the proof is complete.

TheOrem 4.2. Assume (i)-(v) and $\left(\mathrm{vi}^{\prime}\right)$ or $\left(\mathrm{vi}^{\prime \prime}\right)$. Then there exists a (unique) solution $u \in W_{2}^{1} \cap C(\bar{D})$ of the problem (1.5). Moreover, $u \in$ $\mathcal{W}_{\alpha \vee 0}(x)$ for each $x \in D$ and $\sup _{x \in D}\left\|F_{u}\right\|_{\alpha \vee 0 ; x}<\infty$ with $\alpha=0$ in the first case and any $\alpha$ satisfying condition ( $\left.\mathrm{vi}^{\prime \prime}\right)$ in the second one.

Proof. By Remark 2.1 we may and will assume that $\alpha>0$. Throughout the proof $\gamma=\alpha$ if $b=0$ and $2 \mu+L^{2}>0$, and $\gamma=\alpha_{0} / q^{\prime}$ if $2 \mu+L^{2} \leq 0$, where $\alpha_{0}$ is the constant from Remark 2.1 and $q^{\prime}$ is the Hölder conjugate of some $q \in(1, p /(2 \vee d))$. Moreover, we write $\alpha_{1}=\delta+2 \mu+(1+\varepsilon) L^{2}$, where $\delta, \varepsilon>0$ are chosen so that $\alpha_{1}<\gamma, \delta+\varepsilon L^{2}<\alpha_{0} / 2$ and $\varepsilon L^{2}\left\|G_{D} 1\right\|_{\infty}<1$.

The proof will be divided into 5 steps.

Step 1. We first prove that for any square-integrable $w: D \rightarrow \mathbb{R}^{d}$ such that $\sup _{x \in D}\|w\|_{\mathcal{W}_{\gamma}(x)}<\infty$ the problem

$$
A v=-\widetilde{F}_{v},\left.\quad v\right|_{\partial D}=\varphi,
$$

where $\widetilde{F}_{v}(x)=\widetilde{f}(x, v(x)), \widetilde{f}(x, y)=f(x, y, w(x)), x \in D, y \in \mathbb{R}$, is uniquely solvable in $W_{2}^{1}$. For this purpose, for $n \in \mathbb{N}$ we define $a_{n}, b_{n}$ by (3.7), and $h_{n}=\left(h_{n}^{1}, \ldots, h_{n}^{d}\right): D \rightarrow \mathbb{R}^{d}$ and $f_{n}: D \times \mathbb{R} \rightarrow \mathbb{R}$ by

$$
h_{n}^{i}(x)=(-n) \vee w^{i}(x) \wedge n, \quad i=1, \ldots, d, \quad f_{n}(x, y)=f\left(x, y, h_{n}(x)\right) .
$$

Let $\left\{\left(X, P_{x}^{n}\right) ; x \in \mathbb{R}^{d}\right\}$ be a diffusion corresponding to $A^{n}$ defined by (3.3). By [4, Theorem 3.4] (see also [8, Theorem 3.1]), for each $x \in D$ there is a unique solution $\left(Y^{x, n}, Z^{x, n}\right)$ of the $\operatorname{BSDE}(\varphi, \widetilde{f})$ associated with $\left(X, P_{x}^{n}\right)$, and moreover, by [4, Lemma 6.2], $Y^{x, n}=v_{n}\left(X^{\tau}\right) P_{x}^{n}$-a.s., where $v_{n}(x)=Y_{0}^{x, n}$ for $x \in D$. We will show that $v_{n}$ is a unique solution of the problem

$$
A^{n} v_{n}=-\widetilde{F}_{n},\left.\quad v_{n}\right|_{\partial D}=\varphi
$$


in $W_{2}^{1}$, where $\widetilde{F}_{n}: D \rightarrow \mathbb{R}, \widetilde{F}_{n}(x)=f_{n}\left(x, v_{n}(x)\right)$. Since $\alpha_{1}<\gamma$, by $(2.4)$ applied to $v_{n}$ we have

$$
\left|v_{n}(x)\right|^{2} \leq\|\varphi\|_{\infty}^{2} E_{x}^{n} e^{\gamma \tau}+2 \delta^{-1}\left(\|g\|_{\gamma ; x}^{2}+L^{2}\|w\|_{\gamma ; x}^{2}\right) .
$$

If $2 \mu+L^{2}>0$ then $\|g\|_{\gamma ; x}^{2} \leq U_{\gamma}(x)\|g\|_{\infty}$, and if $2 \mu+L^{2} \leq 0$ then by Hölder's inequality,

$$
\|g\|_{\gamma ; x}^{2} \leq\left(U_{\gamma q^{\prime}}(x)\right)^{1 / q^{\prime}}\left(\int_{D}\left(G_{D}(x, y)\right)^{(p / 2 q)^{\prime}} d y\right)^{1 /(p / 2 q)^{\prime} q}\|g\|_{p}^{2},
$$

where $(p / 2 q)^{\prime}$ is the Hölder conjugate to $p / 2 q$. By [13, Corollary A.1.2] and [5, Theorem 8.16] (for $d=1$ see remarks at the beginning of Section 3), $\sup _{x \in D}\left\|G_{D}(x, \cdot)\right\|_{r}<\infty$ for any $r>1$ whose Hölder conjugate is greater than $(2 \vee d) / 2$. Hence, in both cases, $\sup _{x \in D}\|g\|_{\gamma ; x}^{2}<\infty$. From this and (4.5) we see that $v_{n} \in \mathbb{L}_{2}$, hence $\widetilde{F}_{n} \in \mathbb{L}_{2}$. Set now $\varphi_{k}=\varphi * j_{k}$ and $\widetilde{F}_{n, k}=((-k) \vee$ $\left.\widetilde{F}_{n} \wedge k\right) * j_{k}$ for $k \in \mathbb{N}$, where $j_{k}$ is defined as in the proof of Theorem 3.7. Since $D$ is regular, $\varphi_{k} \in C^{\beta}(\bar{D})=C^{0, \beta}(\bar{D})$ and $\widetilde{F}_{n, k}$ are bounded and belong to $C^{\beta}(D)$, it follows from [5, Theorem 6.11] that there is a unique solution $v_{n, k} \in C^{2, \beta}(D) \cap C(\bar{D})$ of the problem $A^{n} v_{n, k}=-\widetilde{F}_{n, k},\left.v_{n, k}\right|_{\partial D}=\varphi_{k}$. By Itô's formula, $\left(Y_{t}^{n, k}, Z_{t}^{n, k}\right)=\left(v_{n, k}\left(X_{t}^{\tau}\right),\left(\sigma_{n} \nabla v_{n, k}\right)\left(X_{t}^{\tau}\right)\right), t \geq 0$, is a solution of the $\operatorname{BSDE}\left(\varphi_{k}, \widetilde{F}_{n, k}\right)$ associated with $\left(X, P_{x}^{n}\right)$. Therefore

$$
\begin{aligned}
Y_{t}^{n, k}= & \varphi_{k}\left(X_{\tau}\right)+\int_{t \wedge \tau}^{\tau} \widetilde{F}_{n, k}\left(X_{s}\right) d s \\
& -\int_{t \wedge \tau}^{\tau}\left\langle Z_{s}^{n, k}, \sigma_{n}^{-1}\left(X_{s}\right) d M_{s}^{n}\right\rangle, \quad t \geq 0, P_{x}^{n} \text {-a.s. }
\end{aligned}
$$

Obviously $\varphi_{k} \rightarrow \varphi$ uniformly on $\bar{D}$ and $\widetilde{F}_{n, k} \rightarrow \widetilde{F}_{n}$ in $\mathbb{L}_{2}$ as $k \rightarrow \infty$. Hence, by Proposition $3.3, v_{n, k} \rightarrow \widetilde{v}_{n}$ in $W_{2}^{1}$, where $\widetilde{v}_{n} \in W_{2}^{1}$ is a unique solution of the problem $A^{n} \widetilde{v}_{n}=-\widetilde{F}_{n},\left.\widetilde{v}_{n}\right|_{\partial D}=\varphi$. On the other hand, by (2.6) we have

$$
\left|v_{n, k}(x)\right| \leq\|\varphi\|_{\infty}+\left\|g^{1 / 2} * j_{k}\right\|_{0 ; x}+K\left\|v_{n}\right\|_{\infty}+L n,
$$

since $\left\|\varphi_{k}\right\|_{\infty} \leq\|\varphi\|_{\infty}$ and $\left|\widetilde{F}_{n, k}\right| \leq\left|\widetilde{F}_{n}\right| * j_{k}$. Consequently, $\left\{v_{n, k}\right\}_{k \in \mathbb{N}}$ is bounded in $D$. Using the above properties of $\left\{\varphi_{k}\right\}_{k \in \mathbb{N}},\left\{\widetilde{F}_{n, k}\right\}_{k \in \mathbb{N}},\left\{v_{n, k}\right\}_{k \in \mathbb{N}}$ and the fact that $\widetilde{F}_{n} \in \mathcal{L}_{0}(x)$ for $x \in D$, as in the proof of (3.9) we deduce from (4.6) that there is a continuous version $\bar{v}_{n}$ of $\widetilde{v}_{n}$ such that

$$
\bar{v}_{n}\left(X_{t}\right)=\varphi\left(X_{\tau}\right)+\int_{t \wedge \tau}^{\tau} \widetilde{F}_{n}\left(X_{s}\right) d s-\int_{t \wedge \tau}^{\tau}\left\langle\nabla \bar{v}_{n}\left(X_{s}\right), d M_{s}^{n}\right\rangle, \quad t \geq 0,
$$

$P_{x}^{n}$-a.s. At the same time, 


$$
Y_{t}^{x, n}=\varphi\left(X_{\tau}\right)+\int_{t \wedge \tau}^{\tau} \widetilde{F}_{n}\left(X_{s}\right) d s-\int_{t \wedge \tau}^{\tau}\left\langle Z_{s}^{x, n}, \sigma_{n}^{-1}\left(X_{s}\right) d M_{s}^{n}\right\rangle, \quad t \geq 0
$$

$P_{x}^{n}$-a.s. Putting

$$
\xi_{t}=Y_{t}^{x, n}-\bar{v}_{n}\left(X_{t}\right), \quad N_{t}=\int_{0}^{t}\left\langle Z_{s}^{x, n}-\left(\sigma_{n} \nabla \bar{v}_{n}\right)\left(X_{s}\right), \sigma_{n}^{-1}\left(X_{s}\right) d M_{s}^{n}\right\rangle
$$

we have $\xi_{t}=N_{t \wedge \tau}-N_{\tau}, t \geq 0$, and hence

$$
\xi_{t}=\xi_{t \wedge \tau}=E_{x}^{n}\left(\xi_{t \wedge \tau} \mid \mathcal{F}_{t \wedge \tau}\right)=E_{x}^{n}\left(-N_{\tau} \mid \mathcal{F}_{t \wedge \tau}\right)+N_{t \wedge \tau}=0 .
$$

Therefore $\bar{v}_{n}\left(X_{t}^{\tau}\right)=Y_{t}^{x, n}, t \geq 0, P_{x}^{n}$ a.s., and consequently $\bar{v}_{n}(x)=\bar{v}_{n}\left(X_{0}^{\tau}\right)$ $=Y_{0}^{x, n}=v_{n}(x)$.

From what has already been proved it follows that $v_{n}$ is a unique, in $W_{2}^{1}$, weak solution of the problem (4.4), and moreover, $\left(v_{n}\left(X^{\tau}\right),\left(\sigma_{n} \nabla v_{n}\right)\left(X^{\tau}\right)\right)$ is a solution of the $\operatorname{BSDE}(\varphi, \widetilde{f})$ associated with $\left(X, P_{x}^{n}\right)$. By the definition of a weak solution, $v_{n}-\varphi \in \dot{W}_{2}^{1}$ and

$$
\begin{aligned}
\left(a_{n} \nabla\left(v_{n}-\varphi\right), \nabla \eta\right)_{2}= & 2\left(\widetilde{F}_{n}, \eta\right)_{2}-\left(a_{n} \nabla \varphi, \nabla \eta\right)_{2}+2\left(\nabla\left(v_{n}-\varphi\right), \eta b_{n}\right)_{2} \\
& +2\left(\nabla \varphi, \eta b_{n}\right)_{2}
\end{aligned}
$$

for any $\eta \in \stackrel{\circ}{W}_{2}^{1}$. Putting $\eta=v_{n}-\varphi$ we obtain

$$
\begin{aligned}
\left(a_{n} \nabla\right. & \left.\left(v_{n}-\varphi\right), \nabla\left(v_{n}-\varphi\right)\right)_{2} \\
\leq & 2\left(\widetilde{F}_{n}-\widetilde{F}_{\varphi}, v_{n}-\varphi\right)_{2}+2\left(\widetilde{F}_{\varphi}, v_{n}-\varphi\right)_{2}+\Lambda\|\nabla \varphi\|_{2} \cdot\left\|\nabla\left(v_{n}-\varphi\right)\right\|_{2} \\
\quad & +2 \Lambda\left\|\nabla\left(v_{n}-\varphi\right)\right\|_{2} \cdot\left\|v_{n}-\varphi\right\|_{2}+2 \Lambda\|\nabla \varphi\|_{2} \cdot\left\|v_{n}-\varphi\right\|_{2} \\
\leq & 2 \mu\left\|v_{n}-\varphi\right\|_{2}^{2}+2\left(\|g\|_{2}+K\|\varphi\|_{2}+L\|w\|_{2}\right) \cdot\left\|v_{n}-\varphi\right\|_{2} \\
& +\lambda^{-1} \Lambda^{2}\|\nabla \varphi\|_{2}^{2}+4 \lambda^{-1} \Lambda^{2}\left\|v_{n}-\varphi\right\|_{2}^{2}+2^{-1} \lambda\left\|\nabla\left(v_{n}-\varphi\right)\right\|_{2}^{2} \\
& +2 \Lambda\|\nabla \varphi\|_{2} \cdot\left\|v_{n}-\varphi\right\|_{2},
\end{aligned}
$$

and hence $\left\|\nabla\left(v_{n}-\varphi\right)\right\|_{2} \leq C\left(\|g\|_{2}+\|\varphi\|_{W_{2}^{1}}+\|w\|_{2}+\left\|v_{n}-\varphi\right\|_{2}\right)$ for some $C=C(\lambda, \Lambda, \mu, K, L)$. From this and (4.5) we see that $\left\{v_{n}-\varphi\right\}_{n \in \mathbb{N}}$ is bounded in $\stackrel{\circ}{W}_{2}^{1}$, and therefore, by Rellich's theorem (see, e.g., [5, Theorem 7.22]), precompact in $\mathbb{L}_{2}$. Suppose that for some subsequence (still denoted by $n$ ) $v_{n} \rightarrow v$ in $\mathbb{L}_{2}$. Then $\widetilde{F}_{v_{n}} \rightarrow \widetilde{F}_{v}$ in $\mathbb{L}_{2}$ by [6, Theorem 2.1]. From this and Proposition 3.3 we conclude that $v$ is a weak solution of (4.3).

Step 2. There exists a version $u$ of $v$ such that $u$ is continuous on $\bar{D}$, $\sup _{x \in D}\|u\|_{\mathcal{W}_{\gamma}(x)}<\infty$ and for each $x \in D$ the pair (1.8) is a solution, in $\mathcal{H}_{\gamma ; x}^{1+d}$, of the $\operatorname{BSDE}(\varphi, \widetilde{f})$ associated with $\left(X, P_{x}\right)$. Indeed, by Lemma 3.2 and (4.5), $\sup _{x \in D}\|v\|_{0 ; x}<\infty$. Hence $\sup _{x \in D}\left\|\widetilde{F}_{v}\right\|_{0 ; x}<\infty$, and so, by Theorem 3.7, there is a unique solution $u \in W_{2}^{1} \cap C(\bar{D})$ of $(3.1)$ with $F$ replaced by $\widetilde{F}_{v}$. Since the problem $(3.1)$ is uniquely solvable in $W_{2}^{1}$, it follows that 
$u=v$ a.e. Moreover, again by Theorem 3.7, the pair (1.8) solves the BSDE $(\varphi, \widetilde{f})$ in $\mathcal{H}_{0 ; x}^{1+d}$. Since $(2.2)$ is satisfied and $\sup _{x \in D}\|\widetilde{f}(\cdot, 0)\|_{\gamma ; x}<\infty$, it follows from Proposition 2.3 that $u$ is in fact a solution of class $\mathcal{H}_{\gamma ; x}^{1+d}$ for every $x$ and its norms in $\mathcal{H}_{\gamma ; x}^{1+d}$ are bounded uniformly in $x \in D$.

Step 3. There is a weak solution $u \in W_{2}^{1}$ of (1.5). To prove this, denote by $V$ the space $W_{2}^{1}$ equipped with the norm

$$
\|u\|_{V}^{2}= \begin{cases}\|u\|_{2}^{2}+\left(\alpha-\alpha_{1}\right)\left(u, u U_{\alpha} 1\right)_{2}+\left(a \nabla u, U_{\alpha} 1 \nabla u\right)_{2} & \text { if } b=0, \\ \|u\|_{2}^{2}+\left(a \nabla u, \widehat{G}_{D} 1 \nabla u\right)_{2} & \text { if } b \neq 0,\end{cases}
$$

and let $V_{\gamma}=\left\{u \in V: \sup _{x \in D}\|u\|_{\mathcal{W}_{\gamma}(x)}<\infty\right\}$. Define the mapping $\Phi: V_{\gamma} \rightarrow V$ by letting $\Phi(\widetilde{w})$ be the solution of (4.3) with $w=\sigma \nabla \widetilde{w}$. By what has already been proved, $\Phi$ is well defined and in fact $\Phi: V_{\gamma} \rightarrow V_{\gamma}$. Set $\Phi\left(w_{i}\right)=v_{i}$, $i=1,2, v=v_{1}-v_{2}, w=w_{1}-w_{2}$ and $F=f\left(\cdot, v_{1}, \sigma \nabla w_{1}\right)-f\left(\cdot, v_{2}, \sigma \nabla w_{2}\right)$. If $b=0$ then as in the proof of Theorem 4.1 we show that (4.1) holds with $v$ in place of $u$. On the other hand,

$$
\begin{aligned}
2\left(F, v U_{\alpha} 1\right)_{2}= & 2\left(f\left(\cdot, v_{1}, \sigma \nabla w_{1}\right)-f\left(\cdot, v_{2}, \sigma \nabla w_{1}\right), v U_{\alpha} 1\right)_{2} \\
& +2\left(f\left(\cdot, v_{2}, \sigma \nabla w_{1}\right)-f\left(\cdot, v_{2}, \sigma \nabla w_{2}\right), v U_{\alpha} 1\right)_{2} \\
\leq & 2 \mu\left(v, v U_{\alpha} 1\right)_{2}+2 L\left(|\sigma \nabla w|,\left|v U_{\alpha} 1\right|\right)_{2} \\
\leq & \left(2 \mu+(1+\varepsilon) L^{2}\right)\left(v, v U_{\alpha} 1\right)_{2}+(1+\varepsilon)^{-1}\left(a \nabla w, U_{\alpha} 1 \nabla w\right)_{2},
\end{aligned}
$$

the last inequality being a consequence of an elementary inequality $2 a b \leq$ $\eta a^{2}+\eta^{-1} b^{2}$ with $\eta=(1+\varepsilon) L$. Hence

$$
\begin{aligned}
\|v\|_{2}^{2}+\alpha & \left(v, v U_{\alpha} 1\right)_{2}+\left(a \nabla v, U_{\alpha} 1 \nabla v\right)_{2} \\
& \leq\left(2 \mu+(1+\varepsilon) L^{2}\right)\left(v, v U_{\alpha} 1\right)_{2}+(1+\varepsilon)^{-1}\left(a \nabla w, U_{\alpha} 1 \nabla w\right)_{2} .
\end{aligned}
$$

If $b \neq 0$ then (4.2) holds with $v$ in place of $u$ and, since $2 \mu \leq-L^{2}$, we have

$$
\left(F, v \widehat{G}_{D} 1\right)_{2} \leq \varepsilon L^{2}\left\|\widehat{G}_{D} 1\right\|_{\infty}\|v\|_{2}^{2}+(1+\varepsilon)^{-1}\left(a \nabla w, \widehat{G}_{D} 1 \nabla w\right)_{2} .
$$

Thus, in both cases $\Phi$ is contractive. Set $u_{0}=0, u_{n}=\Phi\left(u_{n-1}\right), n \in \mathbb{N}$. Then $\left\{u_{n}\right\}$ is a Cauchy sequence in $V$ and hence bounded in $\mathbb{L}_{2}$. In fact, it is bounded in $W_{2}^{1}$. Indeed, we have

$$
\begin{aligned}
\lambda\left\|\nabla\left(u_{n}-\varphi\right)\right\|_{2}^{2} \leq & \left(a \nabla\left(u_{n}-\varphi\right), \nabla\left(u_{n}-\varphi\right)\right)_{2} \\
= & -\left(a \nabla \varphi, \nabla\left(u_{n}-\varphi\right)\right)_{2}+2\left(\nabla u_{n},\left(u_{n}-\varphi\right) b\right)_{2} \\
& +2\left(f\left(\cdot, u_{n}, \sigma \nabla u_{n-1}\right), u_{n}-\varphi\right)_{2} \\
\leq & 4^{-1} \lambda\left\|\nabla\left(u_{n}-\varphi\right)\right\|_{2}^{2}+\lambda^{-1} \Lambda^{2}\|\nabla \varphi\|_{2}^{2} \\
& +2^{-1} \lambda\left\|\nabla\left(u_{n}-\varphi\right)\right\|_{2}^{2}+2^{-1} \lambda\|\nabla \varphi\|_{2}^{2}+4 \lambda^{-1} \Lambda^{2}\left\|u_{n}-\varphi\right\|_{2}^{2} \\
& +2\left(g+K\left|u_{n}\right|+L\left|\sigma \nabla u_{n-1}\right|,\left|u_{n}-\varphi\right|\right)_{2} .
\end{aligned}
$$


Hence $\left\|\nabla u_{n}\right\|_{2}^{2} \leq C_{1}+C_{2}\left\|\nabla u_{n-1}\right\|_{2}$ for some $C_{1}, C_{2}$ depending only on $\lambda, \Lambda, K, L,\|g\|_{2}$ and $\sup _{n \geq 0}\left\|u_{n}\right\|_{2}$, which implies boundedness of $\left\{u_{n}\right\}$ in $W_{2}^{1}$. Therefore $\left\{u_{n}\right\}$ is weakly relatively compact in $W_{2}^{1}$. Since we know that $\left\{u_{n}\right\}$ is Cauchy in $V$ as well, it converges in $V$ to some $\widetilde{u}$.

Step 4. $\widetilde{u} \in V_{\gamma}$. To prove this, for fixed $x \in D$ we denote by $\mathcal{V}(x)$ the space $\mathcal{W}_{\gamma}(x)$ equipped with a norm $\|\cdot\|_{\mathcal{V}(x)}$ equivalent to $\|\cdot\|_{\mathcal{W}_{\gamma}(x)}$, defined by

$$
\|u\|_{\mathcal{V}(x)}^{2}=\left(\gamma-\alpha_{1}\right)\|u\|_{\gamma ; x}^{2}+\|\sigma \nabla u\|_{\gamma ; x}^{2} .
$$

Define $\left\{u_{n}\right\}$ as in Step 3. By Step 2, $\Phi: \mathcal{V}(x) \rightarrow \mathcal{V}(x)$ and for each $n \in \mathbb{N}$ the pair $\left(w_{n+1}\left(X^{\tau}\right),\left(\sigma \nabla w_{n+1}\right)\left(X^{\tau}\right)\right)$, where $w_{n}=u_{n}-u_{n-1}$, is a solution, in $\mathcal{H}_{\gamma ; x}^{1+d}$, of the $\operatorname{BSDE}\left(0, F_{n+1}-F_{n}\right)$ with $F_{n}=f\left(\cdot, u_{n}, \sigma \nabla u_{n-1}\right)$. By Itô's formula,

$$
\begin{aligned}
0=e^{\gamma \tau}\left|w_{n+1}\left(X_{\tau}\right)\right|^{2}= & \left|w_{n+1}\left(X_{0}\right)\right|^{2}+\gamma \int_{0}^{\tau} e^{\gamma s}\left|w_{n+1}\left(X_{s}\right)\right|^{2} d s \\
& +\int_{0}^{\tau} e^{\gamma s} w_{n+1}\left(X_{s}\right) d\left(w_{n+1}\left(X_{s}\right)\right)+\int_{0}^{\tau} e^{\gamma s} d\left\langle w_{n+1}\left(X_{.}\right)\right\rangle_{s} .
\end{aligned}
$$

Hence

$$
\begin{aligned}
E_{x} \int_{0}^{\tau} e^{\gamma t}\left\{\gamma\left|w_{n+1}\left(X_{t}\right)\right|^{2}+\left(a \nabla w_{n+1}, \nabla w_{n+1}\right)\left(X_{t}\right)\right\} d t & \\
& \leq 2 E_{x} \int_{0}^{\tau} e^{\gamma t}\left(F_{n+1}-F_{n}\right) \cdot w_{n+1}\left(X_{t}\right) d t .
\end{aligned}
$$

We have

$$
\begin{aligned}
2\left(F_{n+1}-F_{n}\right) \cdot w_{n+1}= & 2\left(f\left(\cdot, u_{n+1}, \sigma \nabla u_{n}\right)-f\left(\cdot, u_{n}, \sigma \nabla u_{n-1}\right)\right) \cdot w_{n+1} \\
= & 2\left(f\left(\cdot, u_{n+1}, \sigma \nabla u_{n}\right)-f\left(\cdot, u_{n}, \sigma \nabla u_{n}\right)\right) \cdot w_{n+1} \\
& +2\left(f\left(\cdot, u_{n}, \sigma \nabla u_{n}\right)-f\left(\cdot, u_{n}, \sigma \nabla u_{n-1}\right)\right) \cdot w_{n+1} \\
\leq & 2 \mu\left|w_{n+1}\right|^{2}+2 L\left|\sigma \nabla w_{n}\right| \cdot\left|w_{n+1}\right| \\
\leq & \left(2 \mu+(1+\varepsilon) L^{2}\right)\left|w_{n+1}\right|^{2}+(1+\varepsilon)^{-1}\left(a \nabla w_{n}, \nabla w_{n}\right) .
\end{aligned}
$$

By the above,

$$
\begin{array}{r}
E_{x} \int_{0}^{\tau} e^{\gamma t}\left\{\left(\gamma-2 \mu-(1+\varepsilon) L^{2}\right)\left|w_{n+1}\left(X_{t}\right)\right|^{2}+\left(a \nabla w_{n+1}, \nabla w_{n+1}\right)\left(X_{t}\right)\right\} d t \\
\leq(1+\varepsilon)^{-1} E_{x} \int_{0}^{\tau} e^{\gamma t}\left(a \nabla w_{n}, \nabla w_{n}\right)\left(X_{t}\right) d t
\end{array}
$$

which shows that $\left\|w_{n+1}\right\|_{\mathcal{V}(x)}^{2} \leq(1+\varepsilon)^{-1}\left\|w_{n}\right\|_{\mathcal{V}(x)}^{2}$. Hence $\left\{u_{n}\right\}$ is a Cauchy sequence in $\mathcal{V}(x)$ and therefore converges to some $u$. In particular, $u_{n} \rightarrow u$ 
in $\mathcal{L}_{0}(x)$. On the other hand, since $u_{n} \rightarrow \widetilde{u}$ in $V, u_{n} \rightarrow \widetilde{u}$ in $\mathbb{L}_{2}$, and hence $u=\widetilde{u}$ a.e. by Lemma 3.2. Consequently, $u_{n} \rightarrow \widetilde{u}$ in $\mathcal{V}(x)$ and

$$
\left\|u_{1}-\widetilde{u}\right\|_{\mathcal{V}(x)} \leq q(1-q)^{-1}\left\|u_{1}-u_{0}\right\|_{\mathcal{V}(x)} \quad \text { with } q=(1+\varepsilon)^{-1 / 2} .
$$

Hence $\|\widetilde{u}\|_{\mathcal{V}(x)} \leq(1-q)^{-1}\left\|u_{1}\right\|_{\mathcal{V}(x)}$, and so $u \in V_{\gamma}$ since $u_{1} \in V_{\gamma}$.

Step 5. By Step $4, \Phi(\widetilde{u})$ is well defined. Therefore $\widetilde{u}$ is a fixed point of $\Phi$, because $\|\widetilde{u}-\Phi(\widetilde{u})\|_{V} \leq\left\|\widetilde{u}-u_{n}\right\|_{V}+\left\|\Phi\left(u_{n-1}\right)-\Phi(\widetilde{u})\right\|_{V}$ for $n \in \mathbb{N}$. Consequently, $\widetilde{u} \in W_{2}^{1}$ is a weak solution of (3.2). Since $F_{\widetilde{u}} \in V_{\gamma}$ it follows from Theorems 3.7 and 4.1 that $\widetilde{u}$ has a version $u \in C(\bar{D})$, which completes the proof.

From the above proof it is evident that if $a \in C^{1, \beta}(\bar{D})$ for some $\beta \in(0,1)$ then in case $b=0$ condition ( vi $\left.^{\prime \prime}\right)$ may be replaced by $\sup _{x \in D} E_{x} e^{\alpha \tau}<\infty$ (in Step 1 we need not approximate $a$ by $\left\{a_{n}\right\}$ ). We do not know whether $\left(\mathrm{vi}^{\prime \prime}\right)$ may be replaced by the latter condition in the case of arbitrary $a$ satisfying (1.2).

Theorem 4.3. Assume (i)-(vi) and define $\alpha$ as in Theorem 4.2. If $u \in$ $W_{2}^{1} \cap C(\bar{D})$ is a solution of (1.5) then for each $x \in D$ the pair (1.8) is a unique, in the class $\mathcal{H}_{\alpha \vee 0 ; x}^{1+d}$, solution of the $\operatorname{BSDE}(\varphi, f)$ associated with $\left(X, P_{x}\right)$.

Proof. This follows from Theorems 3.7 and 4.2.

\section{References}

[1] V. Bally, E. Pardoux and L. Stoica, Backward stochastic differential equations associated to a symmetric Markov process, Potential Anal. 22 (2005), 17-60.

[2] P. Billingsley, Convergence of Probability Measures, Wiley, New York, 1968.

[3] K. L. Chung and Z. Zhao, From Brownian Motion to Schrödinger's Equation, Springer, Berlin, 1995.

[4] R. W. R. Darling and E. Pardoux, Backwards SDE with random terminal time and applications to semilinear elliptic PDE, Ann. Probab. 25 (1997), 1135-1159.

[5] G. Gilbarg and N. S. Trudinger, Elliptic Partial Differential Equations of Second Order, 2nd ed., Springer, Berlin, 1983.

[6] M. A. Krasnosel'skii, Topological Methods in the Theory of Nonlinear Integral Equations, Macmillan, New York, 1964.

[7] A. Lejay, BSDE driven by Dirichlet process and semi-linear parabolic PDE. Application to homogenization, Stochastic Process. Appl. 97 (2002), 1-39.

[8] É. Pardoux, Backward stochastic differential equations and viscosity solutions of systems of semilinear parabolic and elliptic PDEs of second order, in: Stochastic Analysis and Related Topics VI (Geilo, 1996), L. Decreusefond et al. (eds.), Birkhaüser, Boston, 1998, 79-127.

[9] S. Peng, Probabilistic interpretation for systems of quasilinear parabolic partial differential equations, Stochastics Stochastics Rep. 37 (1991), 61-74. 
[10] A. Rozkosz, On Dirichlet processes associated with second order divergence form operators, Potential Anal. 14 (2001), 123-149.

[11] —, Backward SDEs and Cauchy problem for semilinear equations in divergence form, Probab. Theory Related Fields 125 (2003), 393-407.

[12] A. Rozkosz and L. Słomiński, On existence and stability of weak solutions of multidimensional stochastic differential equations with measurable coefficients, Stochastic Process. Appl. 37 (1991), 187-197.

[13] B. Simon, Schrödinger semigroup, Bull. Amer. Math. Soc. (N.S.) 7 (1982), 447-526.

[14] G. Stampacchia, Le problème de Dirichlet pour les équations elliptiques du second ordre à coefficients discontinus, Ann. Inst. Fourier 15 (1965), no. 1, 189-258.

[15] I. L. Stoica, A probabilistic interpretation of the divergence and BSDE's, Stochastic Process. Appl. 103 (2003), 31-55.

[16] D.W. Stroock, Diffusion semigroups corresponding to uniformly elliptic divergence form operators, in: Séminaire de Probabilités XXII, Lecture Notes in Math. 1321, Springer, Berlin, 1988, 316-347.

[17] D. W. Stroock and S. R. S. Varadhan, Multidimensional Diffusion Processes, Springer, New York, 1979.

Faculty of Mathematics and Computer Science

Nicolaus Copernicus University

Chopina $12 / 18$

87-100 Torun, Poland

E-mail: rozkosz@mat.uni.torun.pl

Received February 24, 2003

Revised version March 8, 2005 